Conclusion: Differences in study design and classification criteria result in slightly different patient populations in each registry. The outcome of pregnancies was favourable. Preterm birth rates are within rates reported by the WHO for the EU general population. However, a selection bias of rather planned and well-controlled pregnancies cannot be ruled out. This is the first collaborative analysis of the EuNeP registries. Descriptive data were combined, and will be - as a next step - pooled together.

Funding: This work was supported by a research grant from FOREUM Foundation for Research in Rheumatology.

References:

[1] Giovannopoulou E et al. Curr Rheumatol Rev. 2017;13(3):162-9.

Disclosure of Interests: Yvette Meißner Speakers bureau: Pfizer, Nathalie Costedoat-Chalumeau Grant/research support from: UCB to my institution, Frauke Förger Grant/research support from: Unrestricted grant from UCB, Consultant of: UCB, GSK, Roche, Speakers bureau: UCB, GSK, Doreen Goll: None declared, Anna Moltó Grant/research support from: Pfizer, UCB, Consultant of: Abbvie, BMS, MSD, Novartis, Pfizer, UCB, Rebecca Özdemir: None declared, Marianne Wallenius: None declared, Anja Strangfeld Speakers bureau: AbbVie, BMS, Pfizer, Roche, Sanofi-Aventis, Rebecca Fischer-Betz Consultant of: UCB, Speakers bureau: Abbvie, Amgen, Biogen, BMS, Celgene, Chugai, GSK, Janssen, Lilly, Medac, MSD, Novartis, Roche, UCB, Pfizer.

DOI: 10.1136/annrheumdis-2020-eular.579

\section{FRI0559 \\ ASSOCIATION BETWEEN DIET QUALITY IN CHILDHOOD AND ADULTHOOD AND KNEE SYMPTOMS IN YOUNG ADULTS}

T. Meng ${ }^{1}$, J. Wilson ${ }^{1}$, A. Venn ${ }^{1}$, F. Cicuttini ${ }^{2}$, L. March ${ }^{3}$, M. Cross ${ }^{3}$, T. Dwyer ${ }^{1,4}$, L. Blizzard ${ }^{1}$, G. Jones ${ }^{1}$, L. Laslett ${ }^{1}$, B. Antony ${ }^{1}$, C. Ding ${ }^{1,5} .{ }^{1}$ Menzies Institute for Medical Research, University of Tasmania, Hobart, Australia; ${ }^{2}$ Department of Epidemiology and Preventive Medicine, Monash University, Melbourne, Australia; ${ }^{3}$ Institute of Bone and Joint Research, University of Sydney, Sydney, Australia; ${ }^{4}$ The George Institute for Global Health, University of Oxford, Oxford United Kingdom: ${ }^{5}$ Clinical Research Centre, Zhujiang Hospital, Southern Medical University, Guangzhou, China

Background: Knee osteoarthritis $(\mathrm{OA})$ is the most prevalent joint disease worldwide, but no disease-modifying treatments are available. Existing treatments largely focus on relieving symptoms, but they may have substantial adverse effects. Identifying risk factors affecting knee symptoms is important for developing safer prevention strategies of knee OA symptoms.

Objectives: To describe the associations between diet quality in childhood and adulthood and knee symptoms in young adults.

Methods: Participants were from the Australian Schools Health and Fitness Survey (ASHFS) in 1985, which was conducted to provide benchmark data on the health and fitness of Australian schoolchildren. During 2004-2006, participants were followed up in the Childhood Determinants of Adult Health (CDAH) Study. Dietary measures were collected in ASHFS (aged 10-15 years) and CDAH Study (aged 26-36 years) using food questionnaires. Diet quality was assessed by Dietary Guidelines Index (DGI), reflecting the adherence to Australian Dietary Guidelines. The DGI comprises 9 components and its maximum possible score is 100. A higher score indicated higher diet quality. During 2008-2010, participants (aged 31-41years) were followed up in the CDAH Knee Study. Knee symptoms were collected using Western Ontario and McMaster Universities Osteoarthritis Index (WOMAC). Zero-inflated negative binomial regression analyses were used to assess the associations between diet quality and knee symptoms. Age, sex, body mass index, physical activity, total energy intake, and knee injury history were included as potential confounders based on biological plausibility.

Results: A total of 399 participants (48.4\% were female) were included in analysis. The average childhood and adult DGI was 46.5 and 55.4 , respectively. The prevalence of knee pain, stiffness and dysfunction was $35.1 \%$. $31.6 \%$ and $39.9 \%$, respectively. The overall childhood DGI was not associated with adult knee symptoms. However, the limited intake of discretionary foods in childhood was associated with lower pain (Mean ratio (MR): 0.96, 95\% confidence interval $(\mathrm{Cl})$ : 0.92-1.00) and dysfunction (MR: 0.94, 95\% $\mathrm{Cl}$ : 0.90-0.99). The overall adult DGI was not associated with knee symptoms. However, replacing saturated fats with unsaturated fats in adulthood was associated with lower WOMAC (Pain: MR 0.93, 95\% Cl 0.87-0.99; stiffness: MR 0.93, 95\% Cl 0.87-0.99; dysfunction: MR 0.91, 95\% Cl 0.83-0.99), drinking water in adulthood was associated with lower stiffness (MR: 0.90, 95\% Cl: 0.83-0.99), and fruit intake in adulthood was associated with lower dysfunction (MR: 0.90, 95\% Cl: 0.81-0.99). Moreover, higher DGI score for dairy in adulthood was associated with higher WOMAC (Pain: MR 1.07, 95\%
Cl 1.00-1.13; stiffness: MR 1.13, 95\% Cl 1.05-1.21; dysfunction: MR 1.11, 95\% CI 1.02-1.21). The overall score change of DGI from childhood to adulthood was not associated with adult knee symptoms. However, the score change of replacing saturated fats with unsaturated fats from childhood to adulthood was associated with lower stiffness (MR 0.89, 95\% Cl 0.80-0.98), and the score change of fruit intake was associated with lower dysfunction (MR 0.92 95\% Cl 0.86-0.99)

Conclusion: Several DGI component scores in childhood and adulthood and some changes of DGI component score from childhood to adulthood were associated with knee symptoms in young adults. The results suggested that early-life diet quality may affect knee symptoms in young adults.

Disclosure of Interests: None declared

DOI: 10.1136/annrheumdis-2020-eular.2687

\section{FRI0560 INCREASED RISK OF RHEUMATOID ARTHRITIS DIAGNOSIS IN STATIN USERS IN A LARGE NATIONWIDE US STUDY}

M. Peterson ${ }^{1}$, H. J. Dykhoff ${ }^{1}$, C. S. Crowson ${ }^{2}$, J. M. Davis $I I I^{3}$,

L. Sangaralingham ${ }^{3}$, E. Myasoedova ${ }^{3}$. ${ }^{1}$ Mayo Clinic, Rochester, United

States of America; ${ }^{2}$ Mayo Clinic, Health Sciences Research, Rochester,

United States of America; ${ }^{3}$ Mayo Clinic, Rheumatology, Rochester, United

States of America

Background: Studies evaluating the effect of statin use on the risk of rheumatoid arthritis (RA) onset have shown conflicting results. Most of these studies evaluated European populations while data from the US are scarce.

Objectives: We aimed to assess the association between statin use (and intensity) and RA occurrence using claims data from the US population.

Methods: For this case-control study, we used the OptumLabs Data Warehouse, a large administrative database of commercially insured and Medicare Advantage beneficiaries, to identify cases of RA and matched controls. Cases were defined as patients with 2 or more diagnoses of RA in January 1, 2010 - June 30, 2019 who were $\geq 18$ years old, filled $\geq 1$ prescription for a conventional or biologic disease modifying anti-rheumatic drug, and had no diagnoses of RA during the prior year. Controls were persons without RA matched 1:1 to RA cases on age, sex, census region, calendar year of index date (corresponding to the date of second diagnosis code for RA), and length of prior medical/pharmacy coverage. Statin use was defined as any filled prescription for a statin medication during prior coverage (excluding any new statin prescriptions filled up to 90 days before first RA diagnosis or index date). Logistic regression models were used to estimate odds ratios (OR) with $95 \%$ confidence intervals $(\mathrm{CI})$ adjusted for age, sex, race, census region, calendar year and Charlson comorbidity index (excluding RA component).

Results: The study included 32,465 cases with RA (mean age 57.0, 72.2\% female) and 32,465 matched controls (mean age $57.0,72.2 \%$ female). There were $10,759(33.1 \%)$ statin users among RA patients and $4,016(12.4 \%)$ statin users among the matched controls. Statin use was associated with increased risk of RA (adjusted OR $3.34,95 \% \mathrm{Cl} 3.19-3.49$ ). All levels of statin intensity were associated with increased risk of RA (high OR: $3.60,95 \% \mathrm{Cl} 3.28-3.94$; medium OR: 3.20, 95\% Cl 3.04-3.37; low OR: 3.72, 95\% Cl 3.34-4.15) compared to non-users. Both former and current statin users showed an increased risk of RA (current OR: 3.04, 95\% Cl 2.80-3.30 and former OR: 3.37, 95\% Cl 3.20-3.54) compared to non-users.

Conclusion: This large nationwide study showed increased risk of RA in statin-users vs non-users. The lack of dose dependence may suggest confounding by indication or a common genetic predisposition for cardiovascular disease and RA. The underlying mechanisms for these associations require further investigation.

Disclosure of Interests: Madeline Peterson: None declared, Hayley J. Dykhoff: None declared, Cynthia S. Crowson Grant/research support from: Pfize research grant, John M Davis III Grant/research support from: Research grants from Pfizer, Consultant of: Served on advisory boards for Abbvie and Sanofi-Genzyme, Lindsey Sangaralingham: None declared, Elena Myasoedova: None declared

DOI: 10.1136/annrheumdis-2020-eular.5020

\begin{tabular}{|l|l}
\hline FRI0561 & PRO-INFLAMMATORY DIETS ARE ASSOCIATED \\
WITH INCREASED C-REACTIVE PROTEIN AND \\
RHEUMATOID ARTHRITIS IN THE UK BIOBANK \\
COHORT
\end{tabular}

J. Dainty ${ }^{1}$, E. Sayers ${ }^{2}$, M. Yates ${ }^{1,3}$, A. Macgregor ${ }^{1,4} .{ }^{1}$ Norwich Medical School, Centre for Epidemiology Versus Arthritis, Norwich, United Kingdom; ${ }^{2}$ Quadram Institute, Norwich, United Kingdom; ${ }^{3}$ Ipswich Hospital, Ipswich, 
United Kingdom; ${ }^{4}$ Norfolk \& Norwich University Hospital, Norwich, United Kingdom

Background: Several individual dietary components have been associated with the risk of rheumatoid arthritis (RA) and recent studies have suggested that dietary indices, which account for the consumption of multiple foods, can be used as more complete measures of risk.

Objectives: In this study we aimed to use the Dietary Inflammatory Index (DII), an independent index of dietary variable associated with inflammatory biomarkers, to evaluate potential associations between pro-inflammatory exposures in the diet, an inflammation biomarker (C-reactive protein) and RA onset using the UK Biobank cohort.

Methods: The DII was calculated from data obtained in 24-hour dietary recall questionnaires collected on healthy participants on four separate occasions over an approximate annual period between Feb 2011 and April 2012. Cases of RA in the UK Biobank cohort were identified from the participants with appropriate ICD10 codes and compared against a randomly selected subsample of controls matched (20:1) for age, sex, smoking status and BMI.

Results: Among the 502,519 subjects enrolled in Biobank, 141,769 had completed 24-hour dietary recall questionnaires and had full data for the 18 dietary variables that were required to create the DII (mean $=0.03$, range: $-3.88,4.22$ ). Higher (positive) DII values indicate more pro-inflammatory diets. This index was positively correlated $(p<0.001)$ with $C$-reactive protein (CRP), attesting to the validity of this index for assessing dietary inflammatory potential. A total of 1,423 participants were classified as having RA ( $1 \%$ prevalence in 'dietary' cohort of 141,769 ) according to their ICD10 codes that were last updated in 2018. Their mean age at enrolment (2006-10) was 59 years. There was a significant association between DII and RA: OR 1.05 [1.011.09]; $p=0.028$ ) that suggested RA cases were more likely to be consuming a pro-inflammatory diet.

Conclusion: These data show a significant association between diet, inflammation (CRP) and RA in the UK Biobank population. The findings are consistent with a recent analysis of the US Nurse's Health Study which was based on data only from females, indicating that these findings are likely to be robust and generalisable. Diet is one of the few modifiable factors that has the potential to reduce the risk of future RA onset. These results open the way to providing evidence-based health advice and for designing clinical interventions.

References:

[1] Shivappa N, Steck SE, Hurley TG, Hussey JR, Hebert JR. Designing and developing a literature-derived, population-based dietary inflammatory index. Public health nutrition 2014;17:1689-96.

Acknowledgments: This research has been conducted using the UK Biobank Resource under Application Number ' 33557 '

Disclosure of Interests: None declared

DOI: 10.1136/annrheumdis-2020-eular.5961

\section{FRIDAY, 05 JUNE 2020}

\section{Validation of outcome measures and biomarkers}

\section{FRI0562 THE PERSPECTIVE OF YOUNG PEOPLE WITH INFLAMMATORY ARTHRITIS ON PATIENT REPORTED OUTCOME MEASURES: RESULTS OF A EUROPE-WIDE SURVEY}

A. Alunno ${ }^{1}$, E. Mosor ${ }^{2}$, T. Stamm² ${ }^{2}$ P. Studenic ${ }^{2}$ on behalf of the EULAR

Task Force to develop points to consider (PtC) for including the perspective of young patients with inflammatory arthritis into PROMs. ${ }^{1}$ University of Perugia, Perugia, Italy; ${ }^{2}$ Medical University of Vienna, Vienna, Austria

Background: Although patient-reported outcome measures (PROMs) are widely used in clinical practice and research, it is unclear if these instruments adequately cover the perspective of young people (18-35 years) with inflammatory arthritis (IA). We recently performed focus groups on PROMs with 53 young IA patients from 4 European countries, but the perspective of these patients has never been explored on a large scale.

Objectives: To explore personal experience, opinions and beliefs of young people with IA across Europe concerning PROMs content, characteristics and ways of administration in order to inform EULAR points to consider (PtC) for including the perspective of young patients with IA into PROMs.

Methods: Based on the results of our previous qualitative study, a task force including patients, rheumatologists and health professionals developed an online survey. The survey covered personal experience, preferences and opinions concerning PROMs. After being pilot tested and revised accordingly, the survey was distributed through the EULAR people with arthritis and rheumatism in Europe (PARE), Young PARE networks and the Emerging EULAR Network (EMEUNET).

Results: 547 people ( $88 \%$ females) from 29 countries aged 18-35 years with a diagnosis of juvenile idiopathic arthritis, rheumatoid arthritis, Still's disease, psoriatic arthritis or spondyloarthritis completed the survey (Figure 1). Thirty-seven percent of respondents reported they never filled a PROM. A North-South and West-East Europe gradient was observed $(30.4 \%$ vs $56.3 \%$ and $25.5 \%$ vs $58.8 \%$ respectively). Figure 2 outlines key findings of our survey. Among respondents having filled PROMs $(n=313)$, two thirds perceived their access to PROM results useful for self-management of their health. Discomfort while filling PROMs was an issue for nearly half of the respondents, as questions were perceived as scaring or not relevant. This discomfort, the fear of judgement, or inadequate assessed time frames were major reasons for difficulties in translating the health experience into a rating scale. Still $75 \%$ use their own experience in the past as reference. Among several reasons, people scored differently from what they felt to emphasize how much better or worse they felt from previous assessment. Concerning preferences of numerical rating scales (NRS) or visual analogue scales (VAS) explored in all respondents regardless having ever filled in PROMs, those in favour of VAS mainly reasoned this by having more possibilities to select and those favouring NRS by better readability and interpretation. Maintaining a sitting position, preparing food, doing physical activity, intimacy and sleep problems were the items selected most frequently in the survey as in the qualitative study to be included in PROMs. The implementation of discussion on self-management, education/work and support possibilities at regular clinic visits was considered important by over $60 \%$ of responders. Overall, electronic capturing of PROMs was preferred over paper-based questionnaires $(57 \%$ vs $13 \%)$.

Conclusion: Our survey explored for the first time the personal experience and opinions of young people with IA concerning PROMs on a large scale and confirmed the results obtained in the qualitative study. This survey informed the EULAR PtC for including the perspective of young patients with IA into PROMs.

References:

[1] Mosor E et al. Arthritis Rheumatol. 2019; 71 (suppl 10)

Figure 1: Diagnosis of inflammatory arthritis among respondents
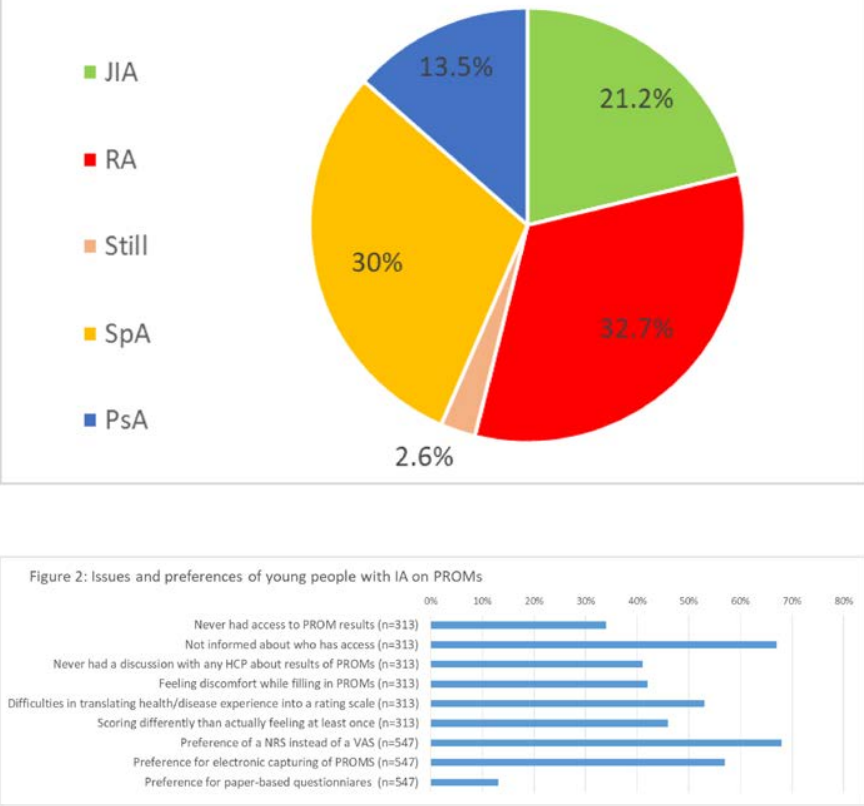

Disclosure of Interests: Alessia Alunno: None declared, Erika Mosor: None declared, Tanja Stamm Grant/research support from: AbbVie, Roche, Consultant of: AbbVie, Sanofi Genzyme, Speakers bureau: AbbVie, Roche, Sanofi, Paul Studenic Grant/research support from: Abbvie DOI: 10.1136/annrheumdis-2020-eular.4618 